\title{
Eigenfrequency optimized 3D continua, with possibility for cavities
}

\author{
Pedersen, Pauli; Pedersen, Niels Leergaard
}

Published in:

Journal of Sound and Vibration

Link to article, DOI:

10.1016/j.jsv.2014.12.024

Publication date:

2015

Document Version

Peer reviewed version

Link back to DTU Orbit

Citation (APA):

Pedersen, P., \& Pedersen, N. L. (2015). Eigenfrequency optimized 3D continua, with possibility for cavities. Journal of Sound and Vibration, 341, 100-115. https://doi.org/10.1016/j.jsv.2014.12.024

\section{General rights}

Copyright and moral rights for the publications made accessible in the public portal are retained by the authors and/or other copyright owners and it is a condition of accessing publications that users recognise and abide by the legal requirements associated with these rights.

- Users may download and print one copy of any publication from the public portal for the purpose of private study or research.

- You may not further distribute the material or use it for any profit-making activity or commercial gain

- You may freely distribute the URL identifying the publication in the public portal

If you believe that this document breaches copyright please contact us providing details, and we will remove access to the work immediately and investigate your claim. 


\title{
Eigenfrequency optimized 3D continua, with possibility for cavities
}

\author{
Pauli Pedersen, Niels L. Pedersen* \\ Department of Mechanical Engineering, Solid Mechanics \\ Nils Koppels Allé, Building 404, DK-2800 Kgs. Lyngby, Denmark
}

\begin{abstract}
Eigenfrequency optimization for 3D continua is formulated and exemplified by the geometry and boundary conditions of a thick plate. Numerical finite element models are based on four node tetrahedra and results from subspace iterations give directly the basis for the continuum redesign. The 3D modeling with a large number of elements has the possibility in optimal design to obtain (as found) not only holes but also cavities in the continuum. Sensitivity analysis is presented on the element level with simple physical interpretation of the involved terms. This general result has general value for control of eigenfrequencies. It is found that in the combination of partial differentiation with the chain rule of differentiation, a specific notation is needed and a suggestion is presented.
\end{abstract}

The optimization method is based on a derived optimality criterion, and as such the maximization problem change to a problem of determining a design with uniform values of this criterion. Non-linear stiffness interpolation may be a physical reality. A two parameter interpolation function is incorporated analytical, also in the sensitivity analysis and the optimality criterion, but without focusing on 1-0 optimal solutions. Two cases of boundary conditions, two cases of total amount of material, and cases of linear and non-linear stiffness interpolation are studied.

Keywords: Eigenfrequency sensitivity, system Rayleigh quotient, local Rayleigh quotient, optimality criterion, recursive optimization, stiffness interpolation

\footnotetext{
* Corresponding author

Email address: nlp@mek.dtu.dk (Niels L. Pedersen)
} 


\section{Introduction}

Optimal design or control of eigenfrequencies and eigenmodes in the design process has many advantages; either in the passive sense for avoiding resonance by moving the eigenfrequencies as far away as possible from an external excitation frequency or

5 e.g. for avoiding the possibility of internal resonance. The active point-of-view is to design structures with specific eigenfrequencies to maximize vibrations i.e. by utilizing the resonance phenomena. Eigenfrequencies can also serve as constraints on optimization of e.g. compliance and or strength, by including a lower bound on the smallest eigenfrequency the risk of ending in a degenerated structure is reduced.

Optimization can normally be performed either using size, shape or topology optimization. The optimization can be performed on the structural level or directly on the continua, and the simplest case is to obtain maximum smallest eigenfrequency. Through half a century, research has been devoted to this subject as listed in the review [1]. The more recent literature presents eigenfrequency optimizations with focus on obtaining clear 1 - 0 (solid - void) optimal designs, i.e., designs without intermediate (gray) densities. Beams, plates and 3D continua are treated and different numerical tools (penalization's) to obtain 1 - 0 designs are applied. Among these papers are [2], [3], [4], [5], [6] and [7]. Note, that 1 - 0 optimal designs is not the goal of the here presented research, that may be classified as traditional size optimization, where stiffness interpolation should be viewed instead from a physical point of view.

The aspects of iteratively redesign for control of eigenfrequency include three steps; analysis, sensitivity analysis and decision of redesign towards a better design. For 3D continua the numerical approaches in these steps may effectively be finite element (FE) analysis combined with subspace iterations in step 1. For step 2 the sensitivity analysis on the system level is simplified as shown by Wittrick [8] and in the present paper it is further simplified down to the element level, resulting in physically interpreted factors. This last point of the sensitivity analysis (gradients) is not well known, but must be of interest also independently of design optimization.

The design is seen as a material distribution in a limited and fixed volume as design 
space. This space is divided into a large number of elements. With design described by the local relative material density in each element, the number of design variables equals the number of elements, say 100.000 as exemplified with a thick plate model. A simple recursive, heuristic redesign is based on a derived optimality criterion, closely related to the analytically obtained derivatives of squared eigenfrequency as a function of changed local material density.

For 3D continua the four node tetrahedron finite element has constant strain, stress and energy density, so an assumption of also constant material density is justified. Then the FE analysis as well as the sensitivity analysis can be based on explicit formula and be performed without numerical integration within each element. Although the sensi-

40 tivity analysis at the system level are presented in [8] with further reference to Jacobi [9], the involved simplicities are not well known outside the optimization community.

With a thick plate as illustrative example in a 3D formulation it must be noted, that extensive results for optimal design of plates are available, mostly based on different 2D theories (simple plate theory, Mindlin plate theory and theory for laminated 45 plates). Stiffness (compliance), strength and eigenfrequency are often taken as objectives. Among many references to the $2 \mathrm{D}$ plate eigenfrequency optimizations a few are listed; an early paper by Olhoff [10], and the eigenfrequency optimization for laminates by Bert [11], that later are followed up by several extensions. Solid plates with ribs are of specific interest and complicated to deal with, an initiating paper is by Cheng and Olhoff [12]. Note, that the plate like example of the present paper is optimized for fixed boundary conditions and fixed outer geometry with uniform thickness. It is therefore rather different from the above mentioned references.

The layout of the paper is as follows. In Section 2 a general model is described as essentially being given by stiffness and mass matrices, and in essence not related to a specific continuum or structural model. In a time independent formulation focus is on elastic energy amplitude and kinetic energy amplitude, both to be accumulated from the respective element quantities. Section 3 comments on the normalized quantities directly obtained from the subspace iteration method, and the specific simplicities for the four node tetrahedron are described in Section 3.1. Section 4 derives the result 60 of the sensitivity analysis, primarily based on stiffness as well as mass direct propor- 
tional to the material density for the element. For the element stiffness this is seldom valid and a more general function of material density is necessary as proportionality factor. Three different functions are discussed, two one parameter functions and a two parameter function, applied here. Simplicity, which is a main focus of the present paper, is obtained even in the extended sensitivity analysis with an interpolation function. Statement of optimization problem and derivation of necessary optimality criterion is presented in Section 5 with a short outline of the applied heuristic numerical procedure for numerical solution in Section 6.

The remaining part of the paper is related to an example, optimizing the small70 est frequency for a thick plate modeled in 3D. Section 7 with data for geometry and material, and with verification of the accuracy of the FE model. General aspects of optimized results are included in Sections 7.2 and 7.3. Final designs after 15 redesigns and corresponding distribution of optimality criterion values are presented in Sections 8 and 9

\section{2. Elastic and kinetic energies on system and element level}

Modeling of a 3D continuum/structure is assumed described by a positive definite, symmetric system stiffness matrix $\mathbf{S}$ and a positive definite, symmetric system mass matrix M. For this model the smallest eigenfrequency $\omega$ and the corresponding eigenmode $\mathbf{d}$ is obtained by the amplitude equilibrium

$$
\mathbf{S d}=\omega^{2} \mathbf{M d}
$$

Simplicity is in focus, so we assume the smallest eigenvalue $\omega^{2}$ to be simple, i.e., non-multiple. For a given design (given $\mathbf{S}$ and $\mathbf{M}$ ) numerical solution to this problem is in a standard setup obtained by the method of subspace iteration, including test of non-multiplicity.

With localized design parameters $\rho_{e}$, the goal is to control $\omega$ and specifically to maximize the smallest $\omega$. For this, the information about the sensitivity, i.e., the gradients $\partial \omega^{2} / \partial \rho_{e}$ for all elements $e$ are needed. To obtain this, the amplitude of total 
elastic energy $U$ is written on system level as well as on local element level.

$$
U=\mathbf{d}^{\mathrm{T}} \mathbf{S d}=\sum_{e} U_{e}=\sum_{e} \mathbf{d}_{e}^{\mathrm{T}} \mathbf{S}_{e} \mathbf{d}_{e}=\sum_{e} \rho_{e} \mathbf{d}_{e}^{\mathrm{T}} \mathbf{S}_{e f} \mathbf{d}_{e}
$$

where the local stiffness matrix at first is assumed proportional to the material density design parameter $\rho_{e}$, with $\mathbf{S}_{e f}$ independent of design. The system stiffness matrix $\mathbf{S}$ may be of order 100000 and the element stiffness matrices $\mathbf{S}_{e}$ are here of order 12. The proportional dependence $\mathbf{S}_{e}=\rho_{e} \mathbf{S}_{e f}$ is later substituted by a more general function $f\left(\rho_{e}\right)$. The fixed matrix $\mathbf{S}_{e f}$ may describe linear elastic isotropy as well as linear elastic anisotropy, but it is not changed during design iterations. Similarly, the amplitude of specific (without factor $\omega^{2} / 2$ ) kinetic energy $T$ is written on system level as well as on element level

$$
T=\mathbf{d}^{\mathrm{T}} \mathbf{M} \mathbf{d}=\sum_{e} T_{e}=\sum_{e} \mathbf{d}_{e}^{\mathrm{T}} \mathbf{M}_{e} \mathbf{d}_{e}=\sum_{e} \rho_{e} \mathbf{d}_{e}^{\mathrm{T}} \mathbf{M}_{e f} \mathbf{d}_{e}
$$

where $\mathbf{M}_{e f}$ is independent of design.

The design parameters $\rho_{e}$ are non-dimensional, positive quantities in the range $0<$ $\rho_{e} \leq 1$ and may be interpreted as relative material volume densities. With $V_{e}$ being the reference volume corresponding to the design parameter $\rho_{e}$ the total amount of a given material volume $V$ is taken as a given quantity, the corresponding constraint equation is

$$
g=\sum_{e} \rho_{e} V_{e}-V=0
$$

The system stiffness matrix $\mathbf{S}$ is the sum of element stiffness matrices $\mathbf{S}_{e}$ in the sense of accumulation according to the FE setup; similarly for the system mass matrix $\mathbf{M}$ and the element mass matrices $\mathbf{M}_{e}$. We write this symbolically

$$
\mathbf{S}=\sum_{e} \mathbf{S}_{e}, \quad \mathbf{M}=\sum_{e} \mathbf{M}_{e}
$$

The non-dimensional design parameter $\rho_{e}$ is local and only has influence on the corresponding $\mathbf{S}_{e}$ and $\mathbf{M}_{e}$. In order to restrict the complexity we throughout the paper assume the influence on the element mass matrix to be simple proportionality

$$
\mathbf{M}_{e}=\rho_{e} \mathbf{M}_{e f}
$$


This is also termed linear interpolation. The influence on the element stiffness matrix is also local and do not change the relations between the individual stiffness components. However, a non-linear interpolation function $f\left(\rho_{e}\right)$ may be the proportionality factor

$$
\mathbf{S}_{e}=f\left(\rho_{e}\right) \mathbf{S}_{e f}
$$

The sensitivity analysis may from an initial look seem complicated but it ends up with a simple formula that contains terms of direct physical interpretation. The derivation involves the need for a specific notation to clarify the partial gradients in connection with the chain rule of differentiation. Earlier, extended index notation has been

85 applied, and having not in the literature located a direct mathematical symbol, we in the present paper suggest a "hat" notation.

The problem of maximizing an eigenfrequency is in the present paper exemplified for a three dimensional (3D) model of a continuum; examples for 2D plane models are presented in [13]. The basic element for the finite element models is a 4 node tetrahedron element, and the accuracy of the FE models are compared to some analytical results. There are interesting aspects of eigenfrequency analysis related to the 3D FE models as compared to 2D plate models. Some comments and verifications are included in the paper, but the main goal of the paper is to communicate the simple and general sensitivity analysis with physical interpretation, and to show specific design optimizations; resulting designs which may include internal cavities.

\section{Analysis by FE subspace iterations}

For a given continuum/structure, analysis by subspace iteration gives a series of modes, described individually by an eigenvector $\mathbf{d}$, orthogonal to the other determined eigenvectors and normalized so that the specific kinetic energy $T=1$. With this normalization of the eigenvector, the specific elastic energy $U$ is numerically equal to the eigenvalue $\omega^{2}$, i.e., for the numerical values $U=\omega^{2}=\omega^{2} T$, where $T$ and $U$ are the time independent amplitudes.

Let us assume an eigenvalue problem described by the symmetric positive definite stiffness matrix $\mathbf{S}$ and the symmetric positive definite mass matrix $\mathbf{M}$. An assumed simple eigenvector (non-multiple eigenvalue) is $\mathbf{d}$ from which the system specific elastic 
energy (twice the strain energy) is $U$ and this energy may be accumulated from element energies $U_{e}$ as stated in (2). Analogously the system specific kinetic energy $T$ may be accumulated from element specific kinetic energies $T_{e}$ as stated in (3)

In the sensitivity analysis we use the following results from analysis

$$
\begin{array}{r}
\omega^{2}, U_{e}, T_{e} \text { with d normalized such that } \\
U=\sum U_{e}=\omega^{2}, T=\sum T_{e}=1, \quad \omega^{2}=U / T
\end{array}
$$

where $U / T$ is the Rayleigh quotient. Further we define element Rayleigh quotients $U_{e} / T_{e}$, that in a somewhat loose notation are termed element squared frequencies

$$
\omega_{e}^{2}=\frac{U_{e}}{T_{e}}=\frac{\mathbf{d}_{e}^{\mathrm{T}} \mathbf{S}_{e} \mathbf{d}_{e}}{\mathbf{d}_{e}^{\mathrm{T}} \mathbf{M}_{e} \mathbf{d}_{e}}
$$

\subsection{Chosen 3D finite element model}

The finite element modeling with an in house program is based on four node tetrahedron elements with constant strain, constant stress and constant energy density, and then analytical expressions can be used to a large extent, see Pedersen [14]. The mesh modeling starts with a brick model and each brick is then in a standard formulation divided into six tetrahedra, as illustrated in Figure 1.

Analysis and design changes are based on the tetrahedron model, but the results (density design and energy densities) may be presented as mean values for the brick of the six corresponding tetrahedra. Two resulting quantities of a tetrahedron are of specific interest for the sensitivity analysis and for the optimality criterion (OC). The element specific elastic energy $U_{e}$, in tetrahedron $e$ follows directly from a displacement mode which give a constant elastic energy density $u_{e}$ and thus

$$
U_{e}=u_{e} \rho_{e} V_{e}
$$

where $V_{e}$ is the geometric reference volume of the tetrahedron element $e$.

The element specific kinetic energy $T_{e}$ in element $e$ need to be determined from the element displacement mode $\mathbf{d}_{e}$ and a consistent mass matrix with no coupling between $x-, y-, z-$ directions. The specific kinetic energy can then be divided into three terms, 

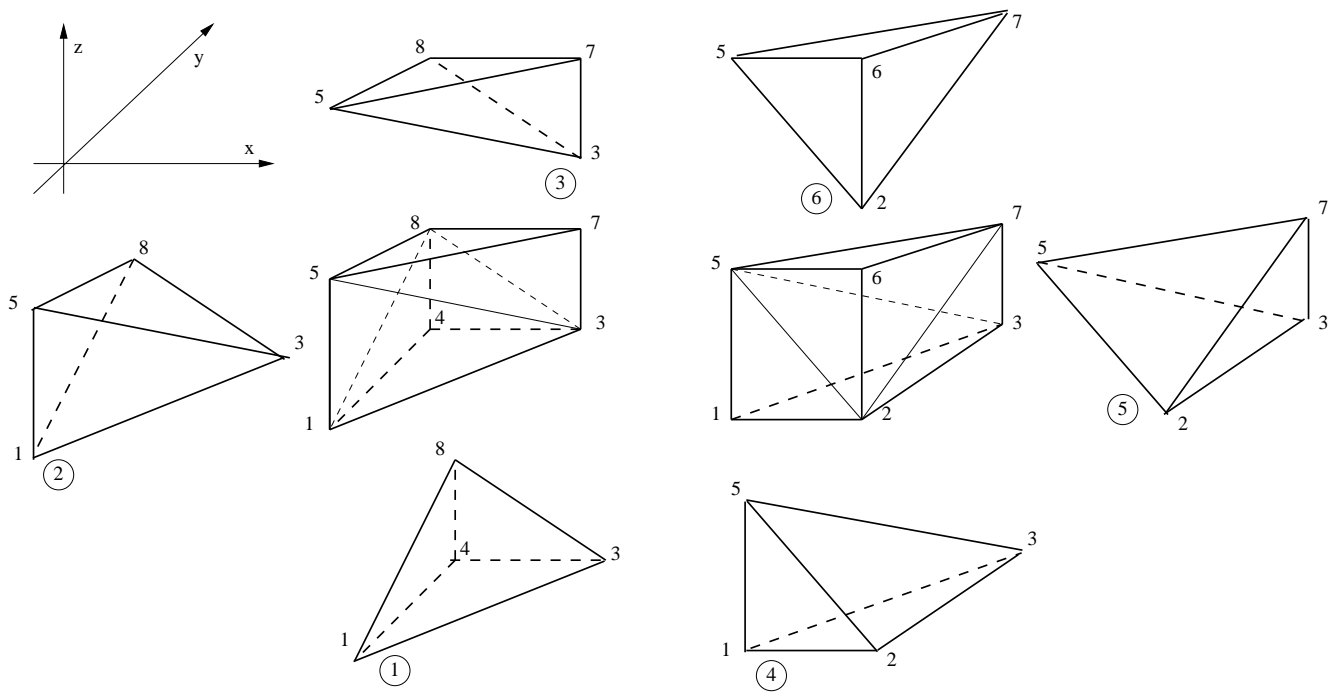

Figure 1: Eight node hexahedron element divided first into two wedges elements and then into six tetrahedra elements, numbered in circles. The numbering of the eight nodes of the hexahedron is also related to the corner nodes of the tetrahedra.

that exemplified for the $x$ - direction is

$$
\left(T_{e}\right)_{x}=\frac{m_{e}}{20} \mathbf{d}_{e x}^{\mathrm{T}}\left[\begin{array}{cccc}
2 & 1 & 1 & 1 \\
1 & 2 & 1 & 1 \\
1 & 1 & 2 & 1 \\
1 & 1 & 1 & 2
\end{array}\right] \mathbf{d}_{e x} \text { with } m_{e}=\rho_{M} \rho_{e} V_{e}
$$

where $m_{e}$ is the mass in element $e$. The mass density with physical dimension $\mathrm{kg} / \mathrm{m}^{3}$ is termed $\rho_{M}$ to distinguish from the non-dimensional material volume densities, that traditionally has the notation $\rho_{e}$. From (11) a rather simple analytical expression follows. In general the FE model is based on explicit formula and no numerical integration is needed.

\section{Sensitivity analysis for squared eigenfrequency}

The design parameters $\rho_{e}$ are assumed to be local, positive non-dimensional quantities in the interval $0<\rho_{e} \leq 1$. With later interpolation extensions we primarily 
assume both the element stiffness matrix $\mathbf{S}_{e}$ and the element mass matrix $\mathbf{M}_{e}$ to be proportional to $\rho_{e}$, i.e.,

$$
\mathbf{S}_{e}=\rho_{e} \mathbf{S}_{e f}, \quad \mathbf{M}_{e}=\rho_{e} \mathbf{M}_{e f}
$$

with both $\mathbf{S}_{e f}$ and $\mathbf{M}_{e f}$ independent of design.

The gradient $\partial \omega^{2} / \partial \rho_{e}=\partial(U / T) / \partial \rho_{e}$ is determined at the element level. To avoid extended indexing a hat notation is introduced by

$$
\frac{\widehat{\partial()}}{\partial \rho_{e}}=\left(\frac{\partial()}{\partial \rho_{e}}\right)_{\text {fixed displacement mode }}=\left(\frac{\partial()}{\partial \rho_{e}}\right)_{\text {fixed strains }}
$$

and with this short notation the gradient is determined, finally at the element level

$$
\frac{\partial \omega^{2}}{\partial \rho_{e}}=\frac{\partial \omega^{2}}{\partial \mathbf{d}} \frac{\partial \mathbf{d}}{\partial \rho_{e}}+\frac{\widehat{\partial \omega^{2}}}{\partial \rho_{e}}=\frac{1}{T^{2}}\left(\frac{\widehat{\partial U}}{\partial \rho_{e}} T-\frac{\widehat{\partial T}}{\partial \rho_{e}} U\right)=\frac{1}{T}\left(\frac{\widehat{\partial U_{e}}}{\partial \rho_{e}}-\omega^{2} \frac{\widehat{\partial T_{e}}}{\partial \rho_{e}}\right)
$$

because $\partial \omega^{2} / \partial \mathbf{d}=\partial(U / T) / \partial \mathbf{d}=\mathbf{0}^{\mathrm{T}}$. This result, based on the assumption of symmetric matrices $\mathbf{S}$ and $\mathbf{M}$, is given by Wittrick [8] with further reference to Jacobi [9]. Inserting the assumptions of linear dependency $\widehat{\partial U_{e} / \partial \rho_{e}}=U_{e} / \rho_{e}$ and $\partial \widehat{T_{e} / \partial \rho_{e}}=$ $T_{e} / \rho_{e}$ gives the local result where the gradient is expressed by local energies

$$
\frac{\partial \omega^{2}}{\partial \rho_{e}}=\frac{1}{T \rho_{e}}\left(U_{e}-\omega^{2} T_{e}\right)=\frac{T_{e}}{T \rho_{e}}\left(\omega_{e}^{2}-\omega^{2}\right)
$$

The gradient is proportional to the difference between the local ratio of energies (local Rayleigh quotient or termed local squared frequency) $\omega_{e}^{2}$ and the system squared eigenfrequency $\omega^{2}$.

From expression (15) follows directly the sign of the gradient as all $T_{e}, T, \rho_{e}$ are non-negative quantities

$$
\frac{\partial \omega^{2}}{\partial \rho_{e}}>0 \text { for } \omega_{e}^{2}>\omega^{2}, \quad \frac{\partial \omega^{2}}{\partial \rho_{e}}<0 \text { for } \omega_{e}^{2}<\omega^{2}, \quad \frac{\partial \omega^{2}}{\partial \rho_{e}}=0 \text { for } \omega_{e}^{2}=\omega^{2}
$$

To increase the frequency of the continuum/structure we increase $\rho_{e}$ for $\omega_{e}^{2}>\omega^{2}$ and decrease $\rho_{e}$ for $\omega_{e}^{2}<\omega^{2}$. A design change may be limited by the volume constraint (4) and by the fact that sensitivity analysis will change when changing the design. The solution to these problems is obtained by the heuristic iterative optimization procedure, shortly described in Section 5 . 


\subsection{Modifications from stiffness non-linear interpolation}

The assumed linear dependence of the element stiffness matrix on the design parameter $\rho_{e}(12)$ may be questioned and an extended analysis is needed and gives the involved modifications. We do not change the assumption of linear dependence for the element mass matrix, but modify (12) to

$$
\mathbf{S}_{e}=f\left(\rho_{e}\right) \mathbf{S}_{e f}, \quad \mathbf{M}_{e}=\rho_{e} \mathbf{M}_{e f}
$$

where $f\left(\rho_{e}\right)$ is an interpolation function, still with $\mathbf{S}_{e f}$ independent of design parameters. From this follows

$$
\frac{\widehat{\partial U_{e}}}{\partial \rho_{e}}=\frac{d f / d \rho_{e}}{f\left(\rho_{e}\right)} U_{e}=\frac{f^{\prime}\left(\rho_{e}\right)}{f\left(\rho_{e}\right)} U_{e}
$$

which for linear interpolation $f\left(\rho_{e}\right)=\rho_{e}$ gives $\partial \widehat{U_{e}} / \partial \rho_{e}=U_{e} / \rho_{e}$ as earlier applied.

With the modification (17) the gradient of the squared eigenfrequency (15) is modified to

$$
\frac{\partial \omega^{2}}{\partial \rho_{e}}=\frac{T_{e}}{T \rho_{e}}\left(\Lambda\left(\rho_{e}\right) \omega_{e}^{2}-\omega^{2}\right) \text { with the definition } \Lambda\left(\rho_{e}\right)=\frac{\rho_{e} f^{\prime}\left(\rho_{e}\right)}{f\left(\rho_{e}\right)}
$$

The conclusions in (16) is modified to

$$
\frac{\partial \omega^{2}}{\partial \rho_{e}}>0 \text { for } \Lambda\left(\rho_{e}\right) \omega_{e}^{2}>\omega^{2}, \frac{\partial \omega^{2}}{\partial \rho_{e}}<0 \text { for } \Lambda\left(\rho_{e}\right) \omega_{e}^{2}<\omega^{2}, \frac{\partial \omega^{2}}{\partial \rho_{e}}=0 \text { for } \Lambda\left(\rho_{e}\right) \omega_{e}^{2}=\omega^{2}
$$

and normally $\Lambda\left(\rho_{e}\right) \geq 1$ for $\rho_{e}>0$.

The non-linear stiffness interpolation is defined by a function $f=f\left(\rho_{e}\right)$ where the function $f$ is the same for all the local density design parameters $\rho_{e}$. A simple polynomial $\rho_{e}^{\kappa_{1}}$ is often applied with the single parameter $\kappa_{1}$, being the slope of $f$ at $\rho_{e}=1$. If $\kappa_{1}$ is three, i.e., $f=\rho_{e}^{3}$, then the interpolation is often termed SIMP. In the present research a two parameter function is applied, with the two parameters $\kappa_{0}, \kappa_{1}$ being the slopes of $f$ at $\rho_{e}=0$ and 1 , respectively.

In the numerical examples we specifically choose $\kappa_{0}=0.1$ and $\kappa_{1}=3$. This function together with the function SIMP is illustrated to the left in Figure 2. The applied function, termed NLPI (Non Linear Penalization or Interpolation), is analytical presented 
in [13] with discussions relative to SIMP and also relative to another one parameter interpolation function, termed RAMP. This function is $f(\rho)=\frac{\rho}{1+\left(\kappa_{1}-1\right)(1-\rho)}$, again with the single parameter being the slope of $f$ at $\rho_{e}=1$.

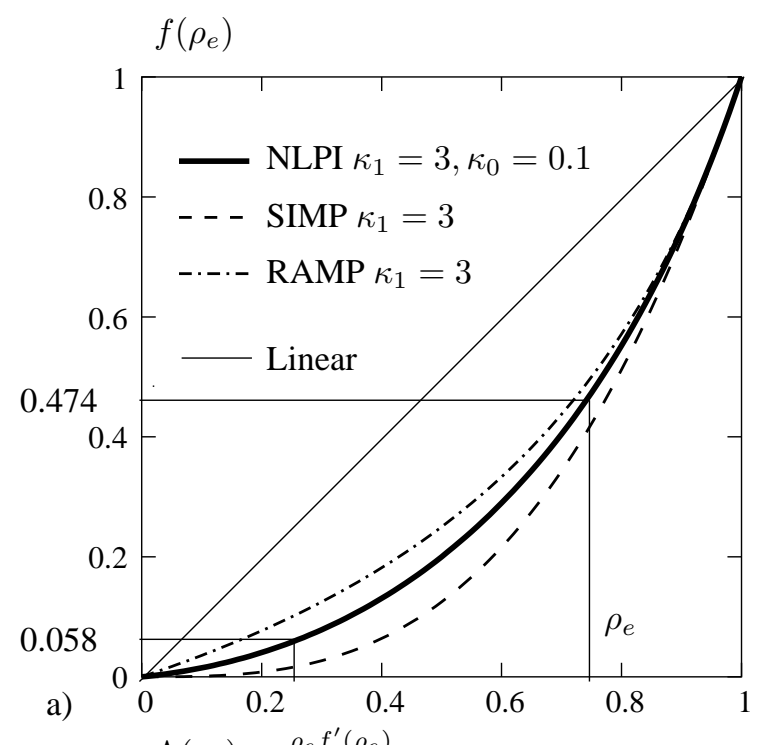
$\Lambda\left(\rho_{e}\right)=\frac{\rho_{e} f^{\prime}\left(\rho_{e}\right)}{f\left(\rho_{e}\right)}$

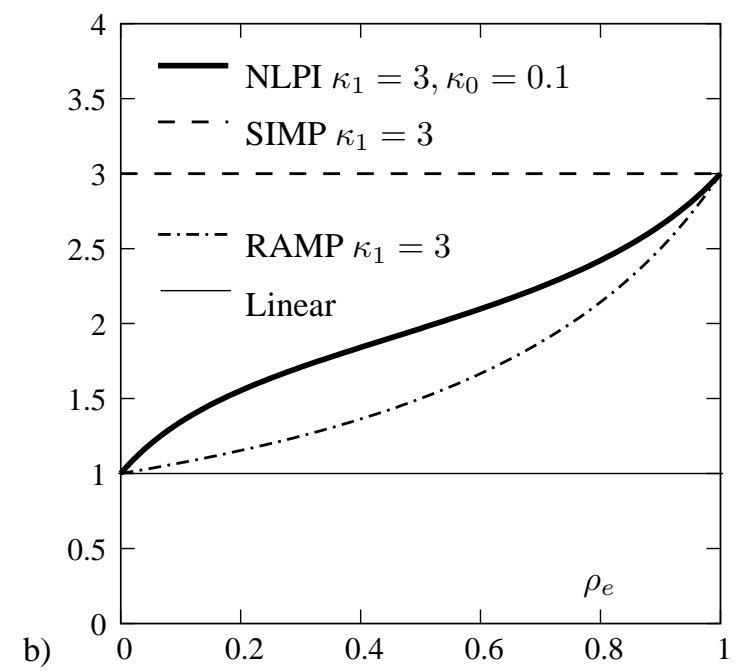

Figure 2: a) The interpolation functions, with the specific values corresponding to $\rho_{e}=0.25$ and $\rho_{e}=0.75$ to be used in Section 7.2. b) The factors for the sensitivity, defined in (19). 


\subsection{Remarks on sensitivities}

The gradients (15) and (19) constitute the basis for an optimization procedure and different possibilities exist. The gradients are important for general control of eigenfrequencies and the simplicity gives a direct physical interpretation:

- The change in squared eigenfrequency $\omega^{2}$, when a local material volume density $\rho_{e}$ is changed, is determined directly by corresponding local quantities from ordinary analysis.

- The gradient is proportional to the local relative kinetic energy amplitude $T_{e} / T$.

- The gradient is inversely proportional to the actual density $\rho_{e}$.

- As stated in (16) and (20) the gradient is proportional to the difference between a weighted local squared frequency (local Rayleigh quotient) and system squared eigenfrequency, i.e., proportional to $\left(\Lambda\left(\rho_{e}\right) \omega_{e}^{2}-\omega^{2}\right)$, with $\Lambda\left(\rho_{e}\right)=1$ for linear proportional stiffness interpolation.

\section{An optimization problem and its optimality criterion}

We study the optimization problem to maximize an eigenvalue (assumed single and being the smallest one) $\omega^{2}$ for a given total amount of material, specified by the volume $V$. We assume this volume constraint to be active and state the problem with non-dimensional densities $\rho_{e}$ as design variables

$$
\begin{aligned}
& \text { Maximize } \omega^{2} \quad \text { (objective) } \\
& \text { for all densities } 0<\rho_{\min } \leq \rho_{e} \leq \rho_{\max } \leq 1 \quad \text { (size limits for design variables) } \\
& \text { and } g=\sum \rho_{e} V_{e}-V=0 \quad \text { (active material constraint) }
\end{aligned}
$$

The OC with only a single, active constraint is proportionality between the gradients of the objective and the gradients of the constraint, i.e.,

$$
\frac{\partial \omega^{2}}{\partial \rho_{e}}=\lambda \frac{\partial g}{\partial \rho_{e}}=\lambda V_{e} \Rightarrow \lambda_{e}=\frac{1}{V_{e}} \frac{\partial \omega^{2}}{\partial \rho_{e}}=\lambda
$$


with the same value $\lambda$ for all elements (sub-domains) $e$ having an active design parameter $\rho_{e}$ where $\rho_{\min }<\rho_{e}<\rho_{\max }$. For a given design a number of different values $\lambda_{e}$ result, and we want to change the design in order for these values to become more equal for the active design elements (resulting in the unknown Lagrange multiplier $\lambda$ ). With linear stiffness interpolation the gradient $\partial \omega^{2} / \partial \rho_{e}$ is given in (15) and the optimality criterion (OC) is

$$
\lambda_{e}=\frac{T_{e}}{T} \frac{1}{\rho_{e} V_{e}}\left(\omega_{e}^{2}-\omega^{2}\right)=\lambda
$$

With stiffness interpolation function $f\left(\rho_{e}\right)$ the gradient $\partial \omega^{2} / \partial \rho_{e}$ is given in (19) and the $\mathrm{OC}$ is

$$
\lambda_{e}=\frac{T_{e}}{T} \frac{1}{\rho_{e} V_{e}}\left(\Lambda\left(\rho_{e}\right) \omega_{e}^{2}-\omega^{2}\right)=\lambda \text { with } \Lambda\left(\rho_{e}\right)=\frac{\rho_{e} f^{\prime}\left(\rho_{e}\right)}{f\left(\rho_{e}\right)}
$$

For illustration a specific functions $\Lambda\left(\rho_{e}\right)$ is graphically included to the right in Figure 2.

\section{Possible heuristic numerical procedure}

The optimization problem (21) is by the OC (24) converted to a problem of finding a continuum of best possible uniformity of the values of the local OC $\left(\lambda_{e}\right)$. Size limits and the active material volume constraint in (4) normally do not allow for satisfying the OC everywhere. Iteratively the active size constraints are fulfilled.

A heuristic procedure is based on the OC, say (24) with (23) as the more simple case for linear stiffness interpolation. A recursive procedure for optimization based on (24) is separated according to the sign of the gradients ( $\operatorname{sign}$ of $\left(\Lambda\left(\rho_{e}\right) \omega_{e}^{2}-\omega^{2}\right)$ ). The redesign of the $\rho_{e}$ follows

For positive gradients $\left(\Lambda\left(\rho_{e}\right) \omega_{e}^{2}-\omega^{2}>0\right)$

$$
\left(\rho_{e}\right)_{\text {new }}=\left(\rho_{e}\right)_{\text {current }}\left(1+4.0 \lambda_{e} / \lambda_{\max }\right)^{0.8} \eta
$$

For negative gradients $\left(\Lambda\left(\rho_{e}\right) \omega_{e}^{2}-\omega^{2}<0\right)$

$$
\left(\rho_{e}\right)_{\text {new }}=\left(\rho_{e}\right)_{\text {current }}\left(1-0.8 \lambda_{e} / \lambda_{\text {min }}\right)^{0.8} \eta
$$


where the values of $\lambda_{\min }<0, \lambda_{\max }>0$ are determined during the evaluation of the gradients. The specific values in (25) 4.0, 0.8, 0.8 are chosen from experience, acting as a kind of move-limits and influence the number of recursive redesigns (number of eigenvalue analysis). The iteratively (without FE analysis) determined volume correction factor $\eta$ relate to the fact that the densities at the limits $\rho_{\min }$ or $\rho_{\max }$ are not known in advance. Factor $\eta$ strictly keep the specified volume by inner iteration where the $\rho_{e}$ at the size limits are localized. The value 0.8 of the power also limits the change of $\rho_{e}$ in one redesign, and such a power (also with a lower value) is often applied for similar recursive procedures. The procedure is applied in the following examples, and have earlier been applied with success to different other problems.

\section{Example with different cases}

The outer geometry (the design domain) of the illustrative example is similar to the thick, rectangular plate shown in Figure 3. Length, width and thickness are $16 \mathrm{~m}, 8 \mathrm{~m}$ and $0.2 \mathrm{~m}$ or $0.4 \mathrm{~m}$. Isotropic material (aluminum) is specified by modulus of elasticity $E=0.7 \cdot 10^{11} \mathrm{~N} / \mathrm{m}^{2}$, Poisson's ratio $\nu=0.3$ and mass density $=2700 \mathrm{~kg} / \mathrm{m}^{3}$. The eigenmode of our interest is assumed double symmetric and thus we can with appropriate boundary conditions restrict the FE modeling to a quarter of the total model. The chosen part is shown in Figure 3 by the full lines.

Dealing with only a quarter of the model a more detailed modeling is possible. Note, that an obtained optimized design may be tested with a total model to make sure that double symmetric eigenmode is the relevant mode. As to multiple eigenmodes, the eigenvalue analysis with the method of subspace iteration give full information, and mode switching during design iteration is therefore directly taken care of. For the treated cases the four smallest eigenfrequencies, corresponding to double symmetric modes, are determined and the two smallest eigenfrequencies are presented for all design iterations. 


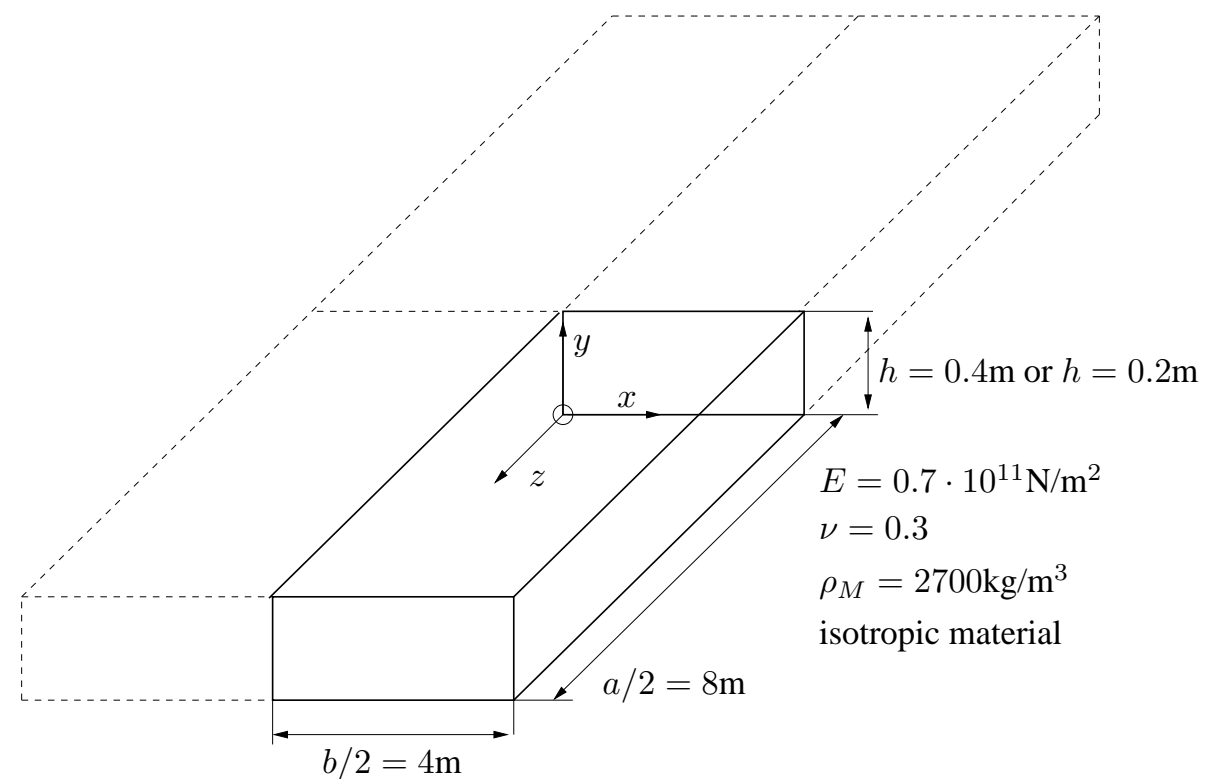

Figure 3: Continuum model, where only the quarter model for analysis and design is shown in full line.

\subsection{Verification of the accuracy of the FE model}

In order to get some knowledge about the accuracy of the obtained eigenfrequencies, a comparison for a specific plate like continuum is performed. The outer dimensions are shown in Figure 3 and simple supports are chosen along the center line of the outer surfaces such that an analytical solution is available, based on thin plate theory and homogeneity. With these most simple assumption a formula of the smallest eigenfrequency with one half wave in the $x$ - as well as in the $z$-direction may be derived to

$$
\left(\omega_{11}\right)_{\mathrm{ss}}=\frac{\pi^{2} h}{\sqrt{8} b^{2}} \sqrt{\frac{E}{\left(1-\nu^{2}\right) \rho_{M}}} \sqrt{1+\left(\frac{b}{a}\right)^{2}}
$$

Inserting the applied parameters: $h=0.2 \mathrm{~m}$ or $0.4 \mathrm{~m}, b=8 \mathrm{~m}, \mathrm{a}=16 \mathrm{~m}, E=7 \cdot 10^{10} \mathrm{~N}$, $\nu=0.3$ and $\rho_{M}=2700 \mathrm{~kg} / \mathrm{m}^{3}$ gives in $\mathrm{Hz}$

$$
\begin{aligned}
& \text { for } h=0.2 m \rightarrow\left(\omega_{11}\right)_{\mathrm{ss}}=10.35 \mathrm{~Hz} \text { and with linear dependence } \\
& \text { for } h=0.4 m \rightarrow\left(\omega_{11}\right)_{\mathrm{ss}}=20.7 \mathrm{~Hz}
\end{aligned}
$$

A FE model with mesh based on equally spaced nodes in $x-, y-, z$-directions equal to 33, 9(in thickness direction) and 65, respectively, implying 19305 nodes 
(57915 d.o.f.), 16384 brick elements, (98304 tetrahedron elements) and bandwidth 897. With homogeneous material, simple supports and linear interpolation, the numerical FE results corresponding to (27) are

$$
\begin{aligned}
& \text { for } h=0.2 m \rightarrow\left(\omega_{11}\right)_{\mathrm{ss}}=9.4 \mathrm{~Hz} \text { and } \\
& \text { for } h=0.4 m \rightarrow\left(\omega_{11}\right)_{\mathrm{ss}}=19.8 \mathrm{~Hz}
\end{aligned}
$$

Both results (27) and (28) are approximations and the differences are found acceptable for our optimization study. Both applied thicknesses must be classified as rather thick plates. The FE results for the two cases show that the linear dependence in (26) for thin plates is no longer valid.

\subsection{Important optimization parameters and}

general aspects of optimized results after 15 redesigns

Three conditions have a major influence on the optimized design and its response. Firstly, the assumed boundary conditions for the continuum, exemplified by simple supported outer boundaries and by clamped outer boundaries, interpreted in relation to the treated quarter model.

Secondly, the amount of available material, specified by the percentages relative to a completely full geometry. This is exemplified by $75 \%$ (large amount of material with less possibility for design changes) and $25 \%$ (less material with large possibilities for design changes). The total amount of material is not subjected to optimization; it is fixed as specified by the equality constraint (4). A parametric study might be the tool to optimize this total amount of material.

Thirdly, how does the local stiffness matrix depend on the local design parameter? This has a strong influence on the optimized design, and results are shown for linear proportional interpolation as well as for non-linear interpolation by a two parameter interpolation function. The local mass matrix is always assumed linear proportional depending on the design parameter. The local stiffness matrices and the local mass matrices are only changed by a factor.

Table 1 shows that with both linear stiffness and linear mass dependence on material density, then the eigenfrequency and the eigenmode is independent of the total 
Table 1: For the continuum with plate geometry, the results (eigenfrequencies in $\mathrm{Hz}$ ) for all treated specific cases; listed for initial homogeneous design as well as for optimized design, all based on $h=0.4 \mathrm{~m}$. Homogeneous design involves equal densities $\rho_{e}$

\begin{tabular}{cccc}
\hline $\begin{array}{c}\text { Boundary } \\
\text { condition }\end{array}$ & $\begin{array}{c}\text { Total } \\
\text { material } \\
\text { volume }\end{array}$ & $\begin{array}{c}\text { Linear stiffness } \\
\text { interpolations } \\
\text { homogeneous } \Rightarrow \text { optimized }\end{array}$ & $\begin{array}{c}\text { Non-linear stiffness } \\
\text { interpolations } \\
\text { homogeneous } \Rightarrow \text { optimized }\end{array}$ \\
\hline Simply & $75 \%$ & $19.8 \mathrm{~Hz} \Rightarrow 25.8 \mathrm{~Hz}$ & $15.7 \mathrm{~Hz} \Rightarrow 23.9 \mathrm{~Hz}$ \\
supported & $25 \%$ & $19.8 \mathrm{~Hz} \Rightarrow 44.4 \mathrm{~Hz}$ & $9.5 \mathrm{~Hz} \Rightarrow 22.5 \mathrm{~Hz}$ \\
\hline Clamped & $75 \%$ & $38.7 \mathrm{~Hz} \Rightarrow 59.1 \mathrm{~Hz}$ & $30.8 \mathrm{~Hz} \Rightarrow 53.4 \mathrm{~Hz}$ \\
supported & $25 \%$ & $38.7 \mathrm{~Hz} \Rightarrow 128.8 \mathrm{~Hz}$ & $18.7 \mathrm{~Hz} \Rightarrow 81.5 \mathrm{~Hz}$ \\
\hline
\end{tabular}

amount of material for a homogeneous continuum ( $75 \%$ or $25 \%$ ), but naturally depending on the outer support. For these two cases the OC values in (23) gives identical distributions for total amount of material equal to $75 \%$ and $25 \%$. Table 1 also shows that for non-linear stiffness interpolation, the eigenfrequencies depend on the total amount of material, also for a homogeneous continuum. From (1) follows for a homogeneous continuum (all densities $\rho_{e}$ assumed equal)

$$
f\left(\rho_{e}\right) \mathbf{S}_{f} \mathbf{d}=\left(\omega_{\rho_{e}}^{2}\right) \rho_{e} \mathbf{M}_{f} \mathbf{d}
$$

where the system matrices $\mathbf{S}_{f}, \mathbf{M}_{f}$ corresponds to full material $\left(\rho_{e}=1\right)$. Thus, the eigenmodes are not changed for homogeneous values of $\rho_{e}$, but the eigenfrequency is changed according to the different factors in (29). With reference to the eigenfrequency for full material $\left(\omega_{f}\right)$ we get

$$
\omega_{\rho_{e} \text { (homogeneous) }}=\sqrt{\frac{f\left(\rho_{e}\right)}{\rho_{e}}} \omega_{f} \text { i.e. with linear } f\left(\rho_{e}\right)=\rho_{e} \Rightarrow \omega_{\rho_{e} \text { (homogeneous) }}=\omega_{f}
$$

The relation between the homogeneous values in Table 1 follows from the relation (30) with the values in Figure 2, i.e., $15.7=\sqrt{0.474 / 0.75} \cdot 19.8, \quad 9.5=\sqrt{0.058 / 0.25}$. $19.8, \quad 30.8=\sqrt{0.474 / 0.75} \cdot 38.7$ and $18.7=\sqrt{0.058 / 0.25} \cdot 38.7$. 
The distribution of values of OC (24) changes, even with unchanged eigenmode,

220 shown for linear interpolation in Figure 4, and therefore not all shown.

Table 1 gives an overview of obtained eigenfrequencies for the in total eight different cases. Hereafter, each case is presented and discussed individually with design history, optimized design and with distributions of $\mathrm{OC}$ values for the corresponding optimized designs.

\subsection{More detailed insights from the examples and limitations}

Behind the choice of the specific example are the following considerations:

- If for a continuum, the distribution of the values of $\mathrm{OC}$ is visualized and shows non-uniformity, then opportunity to change the eigenfrequency corresponding to the actual eigenmode is a possibility. This non-uniformity illustrated in Figure 4 for a homogeneous model with two different support conditions.

- The strong influence from the outer boundary conditions is shown. In Section 8 simple supports are assumed at all outer boundaries, while Section 9 documents the results with clamped outer boundary conditions at all outer boundaries.

- The numerical stability in applying the derived sensitivities and the OC are seen by the histories of eigenfrequencies during redesign, as presented in Figures 5 and 6. The presentations are limited to the two smallest eigenfrequencies, the first one to be maximized in full line while the second one is in broken line. Note, that the quarter FE model assumes double symmetric eigenmodes to be the physical ones of interest.

- The optimized designs after 15 redesigns are shown with their corresponding distributions of the values of OC that within the possible limits are almost uniform in active design areas, in agreement with the necessary condition of optimality. These presentations in terms of nine $x-y$ planes for $z=0,1,2,3,4,5,6,7$ and $8 \mathrm{~m}$. in Section 9. 


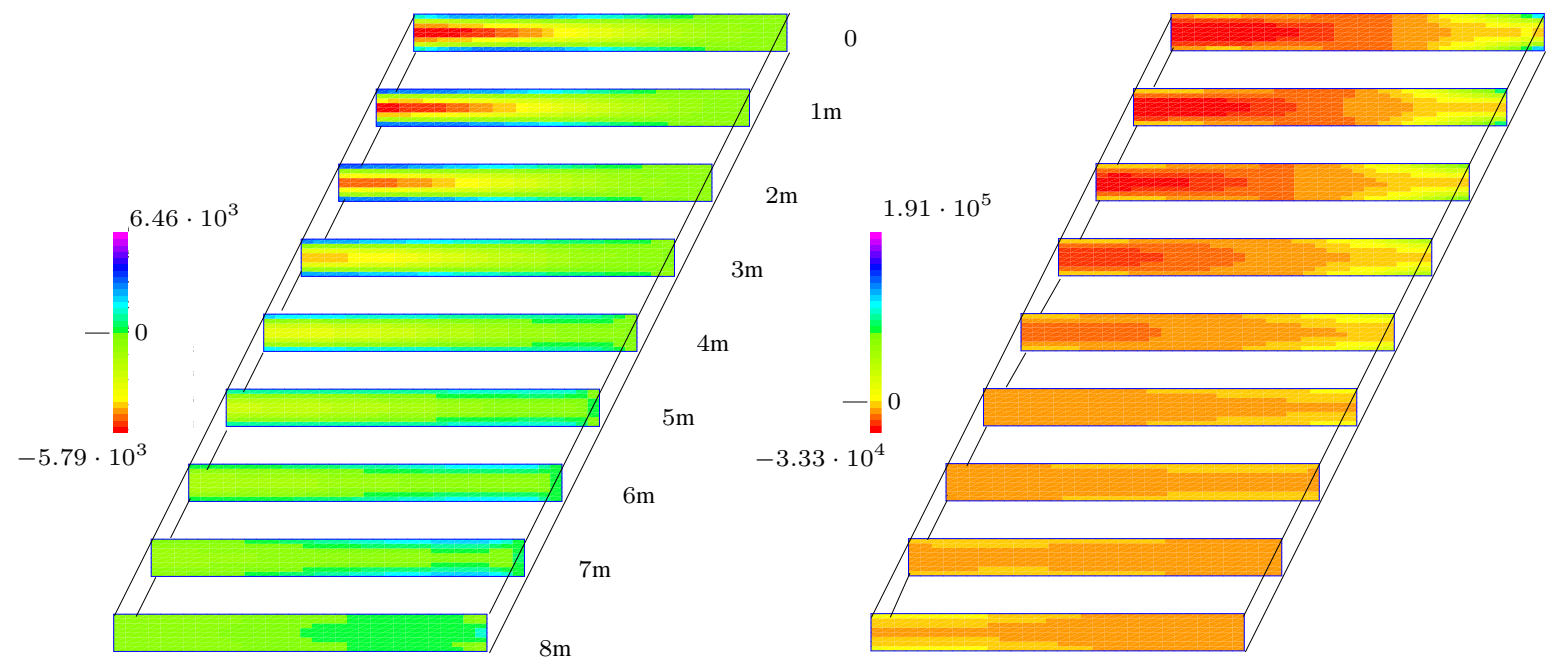

Figure 4: Left the OC values for a homogeneous simple supported model with linear interpolation. Right the OC values when the outer supports are changed to clamped. Linear scales for the OC values.

Figure 4/shows two distributions, both in nine selected $x-y$ planes at $\mathrm{z}=0,1,2,3,4,5,6,7,8 \mathrm{~m}$ of OC values for initial homogeneous designs. Negative values reflects that local material should be removed to increase the smallest eigenfrequency, while positive values reflects that local material should be added to increase the smallest eigenfrequency. In the left figure the initial design is based on simple supports, while the right figure is based on clamped support, both assuming linear interpolation of stiffnesses and masses. As expected the actual boundary conditions are of major importance. As these two OC value distributions are rather different, it may be expected that the optimized designs are different for these two different boundary conditions.

Figure 5 gives a detailed background for the upper half of Table 1. Figure 5 shows no crossings (mode switching) during the redesigns, but in Figure $5 \mathrm{~b}$ this might happen with more redesigns, and the kink of the dotted curve indicate mode switching with a not shown higher order mode. As expected, with less material (Figures 5b and 5d), redesign possibilities are larger and larger increase relative to initial eigenfrequency is possible.

Figure 6 gives a detailed background for the lower half of Table 1. In Figure 6a it is seen that the redesigns procedure for this case stops after 6 redesigns, as all sensitivities 

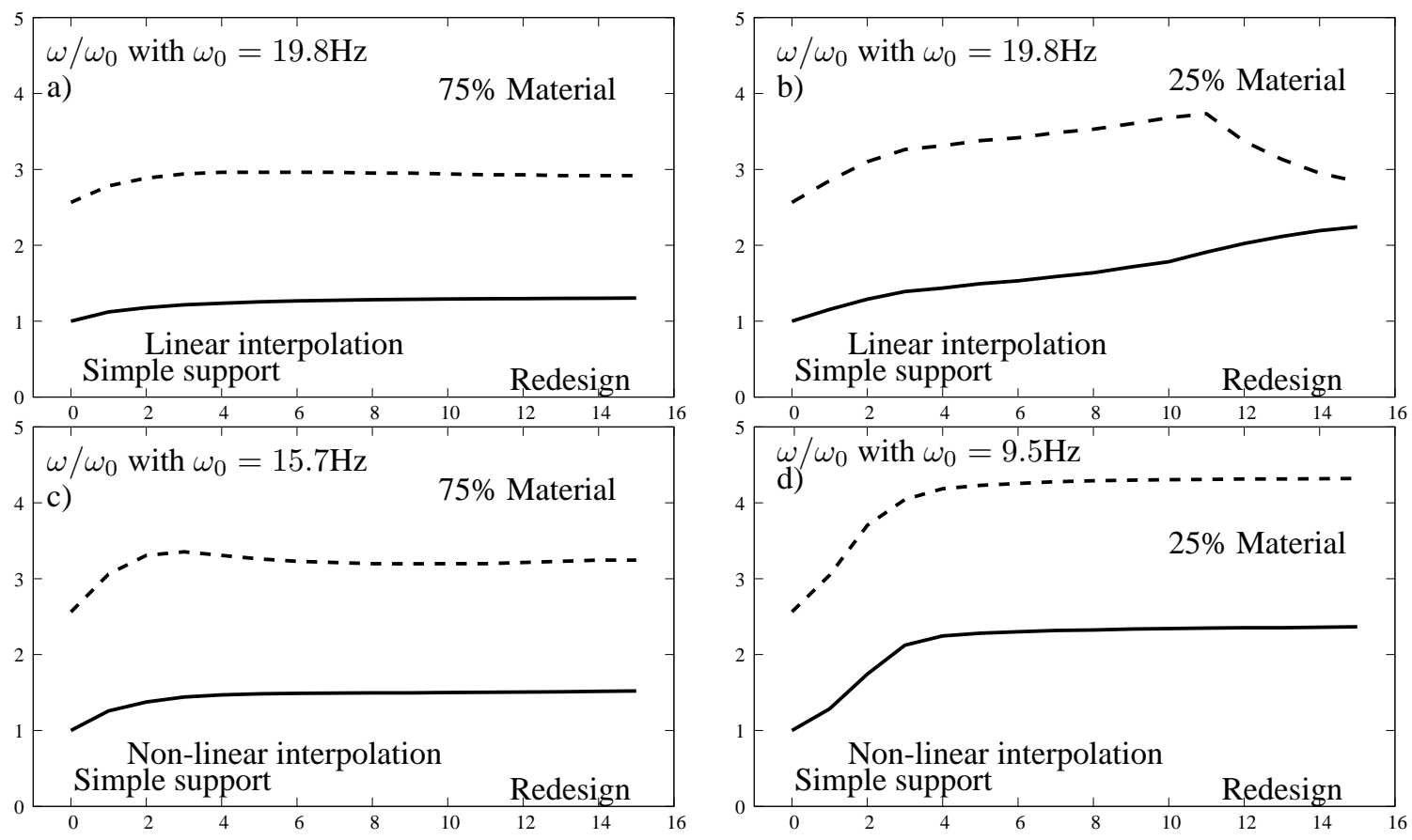

Figure 5: Histories of eigenfrequencies, relative to the smallest eigenfrequency for homogeneous design, for the 2 smallest, double symmetric eigenmodes, during 15 redesigns. The cases of simple supports, $75 \%$ and $25 \%$ material; linear and non-linear interpolation with $\kappa_{1}=3, \kappa_{0}=0.1$. 

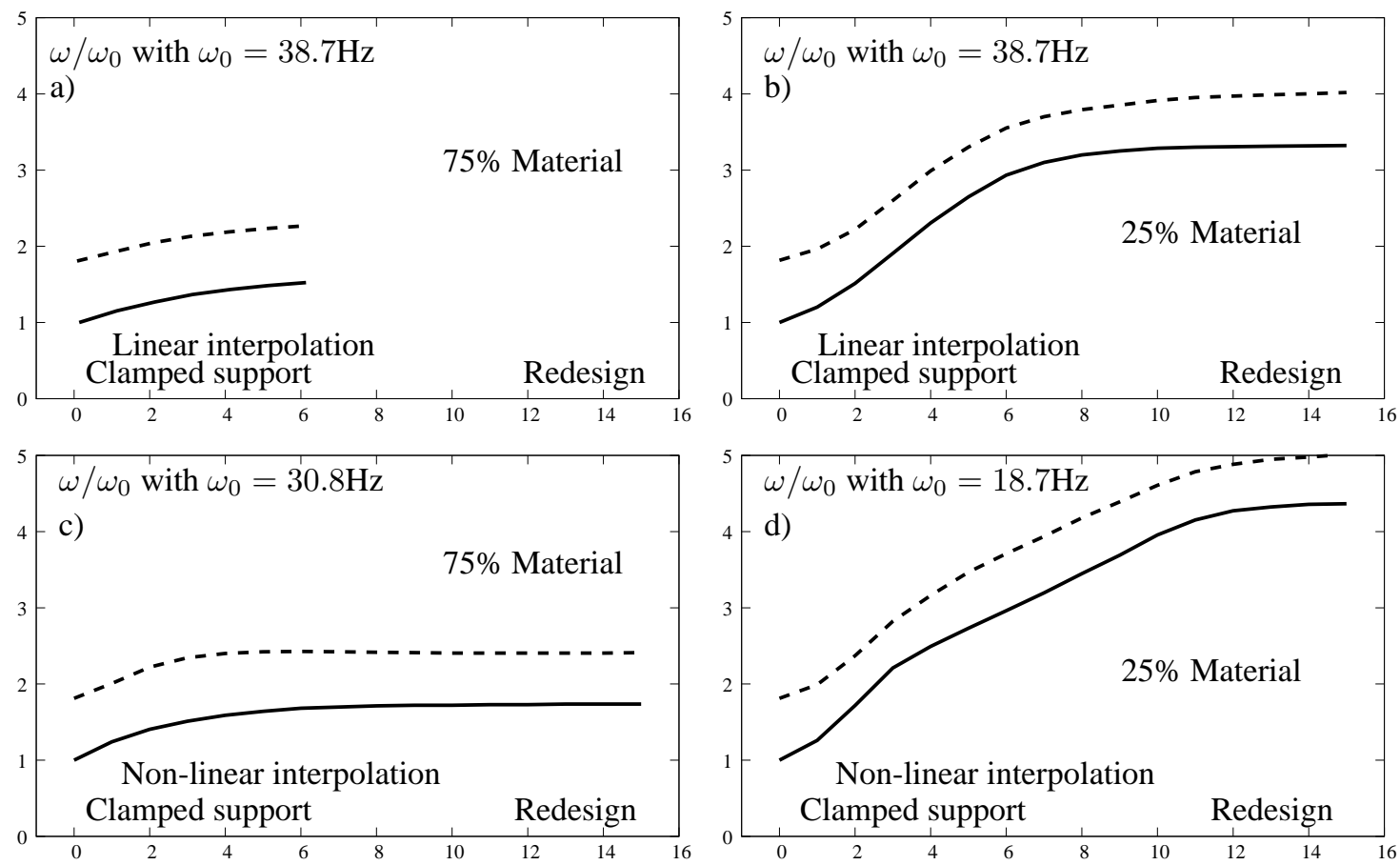

Figure 6: Histories of eigenfrequencies, relative to the smallest eigenfrequency for homogeneous design, for the 2 smallest, double symmetric eigenmodes, during 15 redesigns. The cases of clamped supports, $75 \%$ and $25 \%$ material; linear and non-linear interpolation with $\kappa_{1}=3, \kappa_{0}=0.1$. 
are negative for the active design variables when $75 \%$ material must be used. With non-linear stiffness interpolation the eigenfrequencies are generally lower as it follows from the lower stiffnesses. However, the influence from redesigns may be stronger and Figures $6 \mathrm{~b}$ and $6 \mathrm{~d}$ shows that this is especially true for the clamped supports. Note, that the drastic improvements seen in Figures $6 \mathrm{~b}$ and $6 \mathrm{~d}$ need to be confirmed without the assumption of double symmetry, as eigenmodes without double symmetry might then have a smaller value.

\section{Design results for simple supports}

In relation to the quarter part model of Figure 3, the boundary conditions on the displacements $d_{z}, d_{x}$ for the assumed double symmetric eigenmodes are

$$
\begin{aligned}
& d_{z}=0 \text { for all nodes of the } x-y \text { plane at } z=0 \\
& d_{x}=0 \text { for all nodes of the } y-z \text { plane at } x=0
\end{aligned}
$$

The additional boundary conditions corresponding to the simple outer supports are

line support $d_{y}=0$ for all nodes of the line $x=b / 2, \quad 0 \leq z \leq a / 2, y=h / 2$

line support $d_{y}=0$ for all nodes of the line $0 \leq x \leq b / 2, z=a / 2, y=h / 2$ 


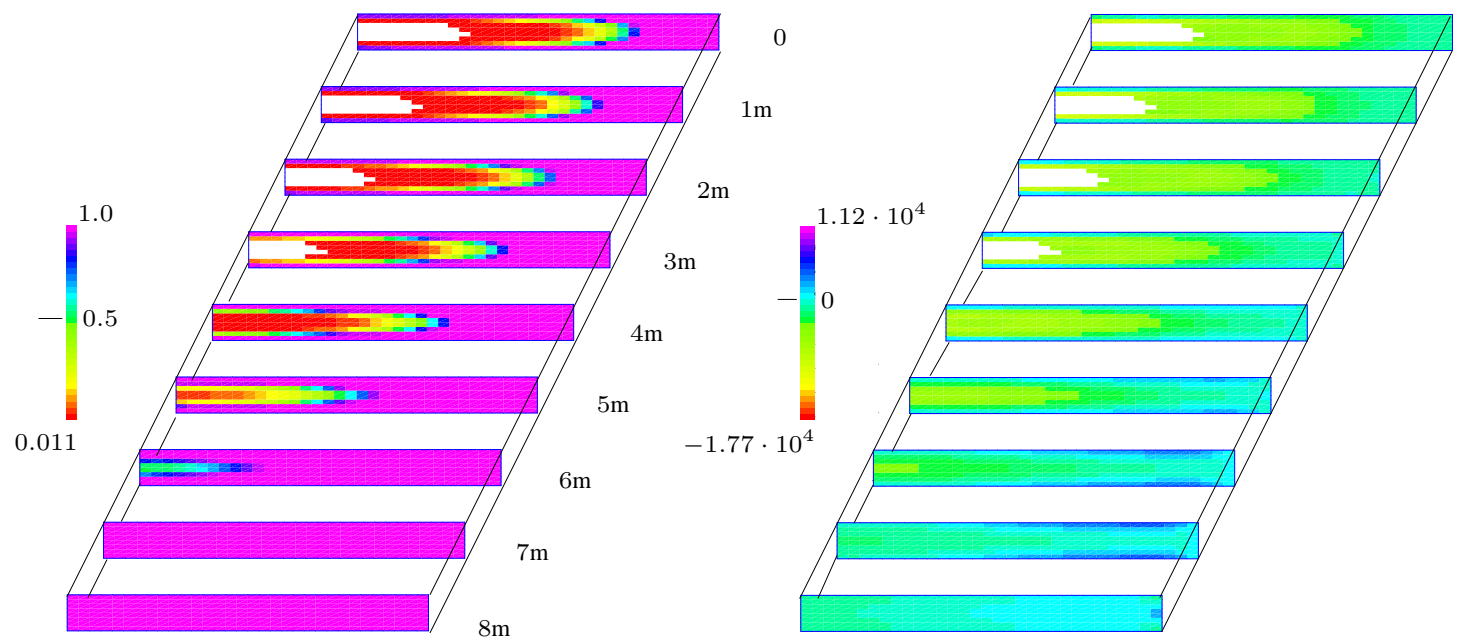

Figure 7: Simple support, linear interpolation and $75 \%$ material. Left the optimized density distribution and right the corresponding distribution of $\mathrm{OC}$ values. Linear scales for density and $\mathrm{OC}$ values; white for $\rho_{e}<0.011$.

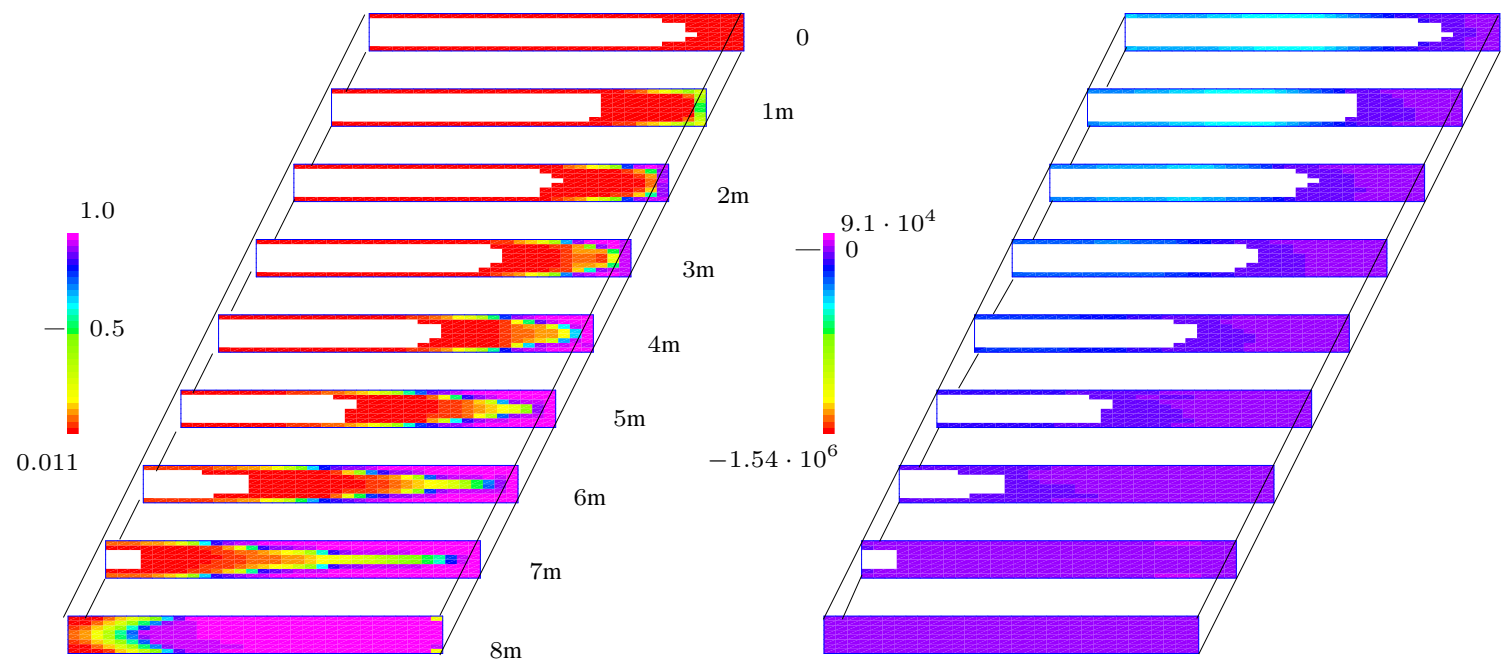

Figure 8: Simple support, linear interpolation and $25 \%$ material. Left the optimized density distribution and right the corresponding distribution of $\mathrm{OC}$ values. Linear scales for density and $\mathrm{OC}$ values; white for $\rho_{e}<0.011$. 


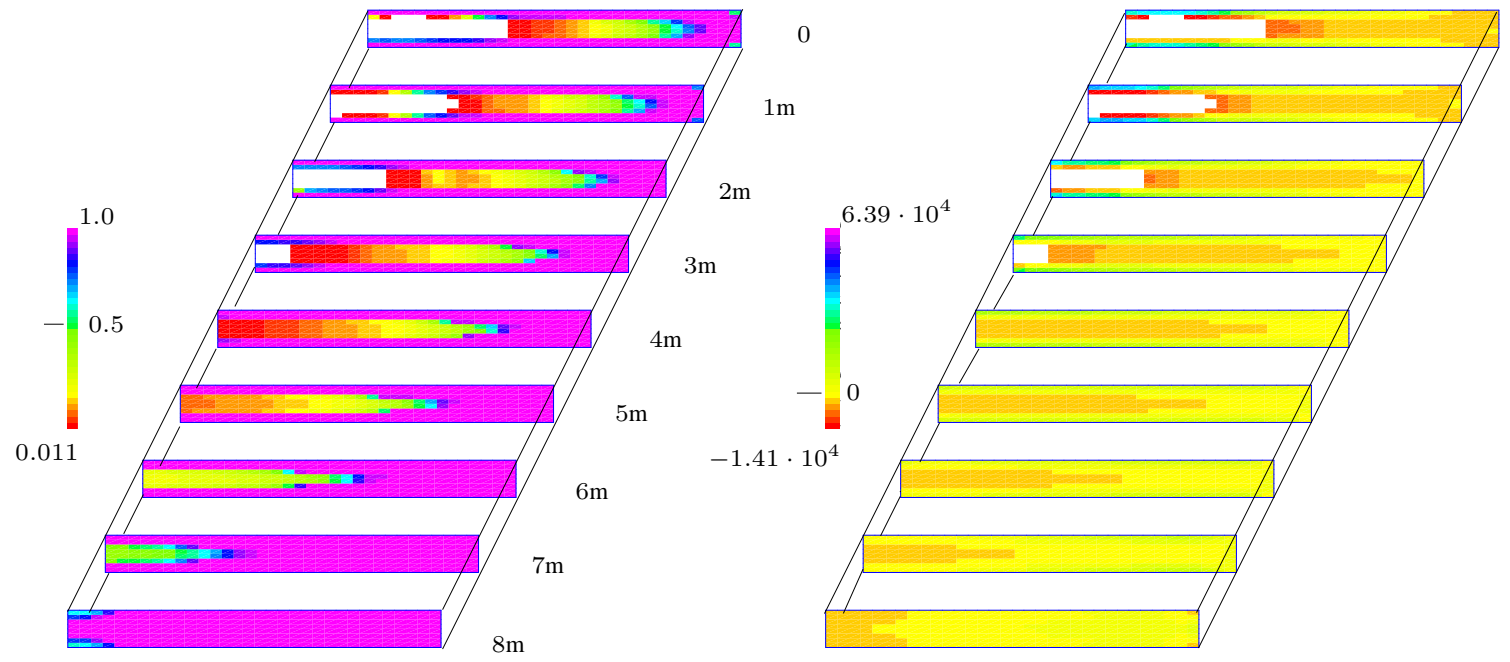

Figure 9: Simple support, non-linear interpolation with $\kappa_{1}, \kappa_{0}=3,0.1$, and $75 \%$ material. Left the optimized density distribution and right the corresponding distribution of OC values. Linear scales for density and $\mathrm{OC}$ values; white for $\rho_{e}<0.011$.

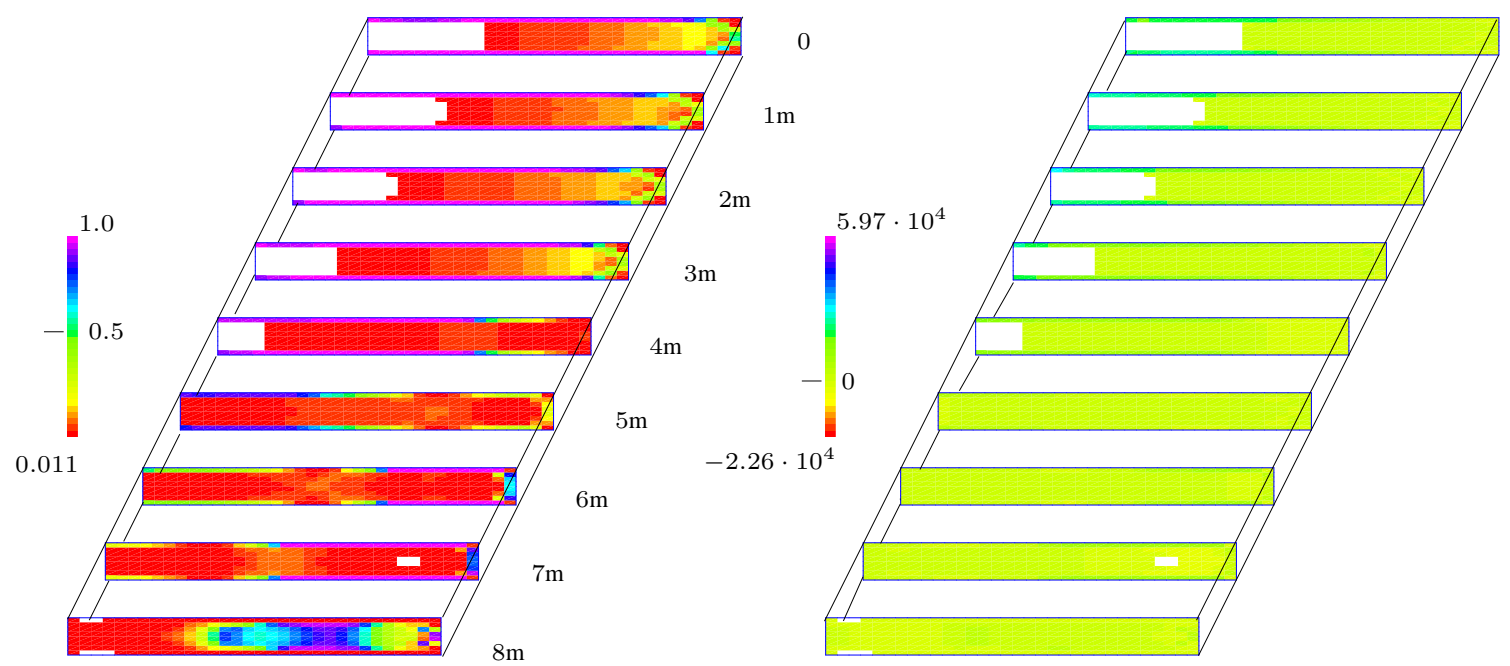

Figure 10: Simple support, non-linear interpolation with $\kappa_{1}, \kappa_{0}=3,0.1$, and 25\% material. Left the optimized density distribution and right the corresponding distribution of OC values. Linear scales for density and $\mathrm{OC}$ values; white for $\rho_{e}<0.011$. 
The comments to the results for simple supports are as follows:

- All Figures 7 - 10 show two distributions in the nine selected $x-y$, planes for $z=0,1,2,3,4,5,6,7,8 \mathrm{~m}$. The left part of each figure shows the optimized design after 15 redesigns, shown by its resulting $\rho_{e}$ distribution. The right part of each figure shows the $\mathrm{OC}$ values for the corresponding design. Note, that with active size constraints: $\rho_{e}=\rho_{\min }$ may give large negative OC values and $\rho_{e}=\rho_{\max }$ may give large positive $\mathrm{OC}$ values.

- Figure 7 shows the final design with history in Figure 5a. For this design a cavity is seen for $z=0,1,2$ and $3 \mathrm{~m}$ in the upper left corner (middle of the total plate). Note, that rather uniform values (green) of OC $\left(\lambda_{e}\right)$ are obtained except for domains with active upper constraint, i.e. domains of $\rho_{e}=1$. Positive OC values (blue) are found in these areas of full material (close to the outer support). The white areas correspond to $\rho_{e}=\rho_{\min }=0.01$, i.e., holes or cavities, where the values of density as well as OC are not shown either. This comment is valid for all resulting figures.

- Figure 8 shows the final design with history in Figure 5 b. Figure 8 is similar to Figure 7 above, except that the total amount of available material is now only $25 \%$. A seen in Figure $5 \mathrm{~b}$ the redesign is not yet converged in 15 redesigns, still a clear result is obtained. Note the extension of the cavity in the middle of the total plate, here the upper left corner. The resulting distribution for the values of $\mathrm{OC}$ is uniform, positive and close to zero. The thickness of the outer layers for the cavity is related to the modeling with nine layers in the thickness direction. Note, that the scales for the many distributions of values of OC are specifically related to the individual figures.

- Figure 9 shows the final design with history in Figure 5 c. Figure 9 is similar to Figure 7, but now the stiffness interpolation is changed from linear to non-linear with the chosen parameters $\kappa_{1}, \kappa_{0}=3,0.1$. A cavity like in Figure 7, and rather uniform values of $\mathrm{OC}$ that are close to zero and negative (orange) in active design domains and positive (green) in domains of full material. 
- Figure 10 shows the final design with history in Figure 5 d. Figure 10 is similar to Figure 9 above, except that the total amount of available material is now only $25 \%$. The cavity is sharp, as expected with non-linear stiffness interpolation. The obtained values of OC distribution rather uniform, close to zero and negative. Positive OC values (darker green) found for the areas of full material. The nonlinear interpolation of stiffness implies more sensitive results.

\section{Design results for clamped supports}

In relation to the quarter part model in Figure 3, the boundary conditions for the assumed double symmetric eigenmodes are unchanged specified by (31). The additional boundary conditions corresponding to the clamped outer supports are

$d_{x}, d_{y}, d_{z}=0,0,0$ for all nodes of the y-z plane $x=b / 2,0 \leq y \leq h, 0 \leq z \leq a / 2$ $d_{x}, d_{y}, d_{z}=0,0,0$ for all nodes of the x-y plane $0 \leq x \leq b / 2,0 \leq y \leq h, z=a / 2$ 


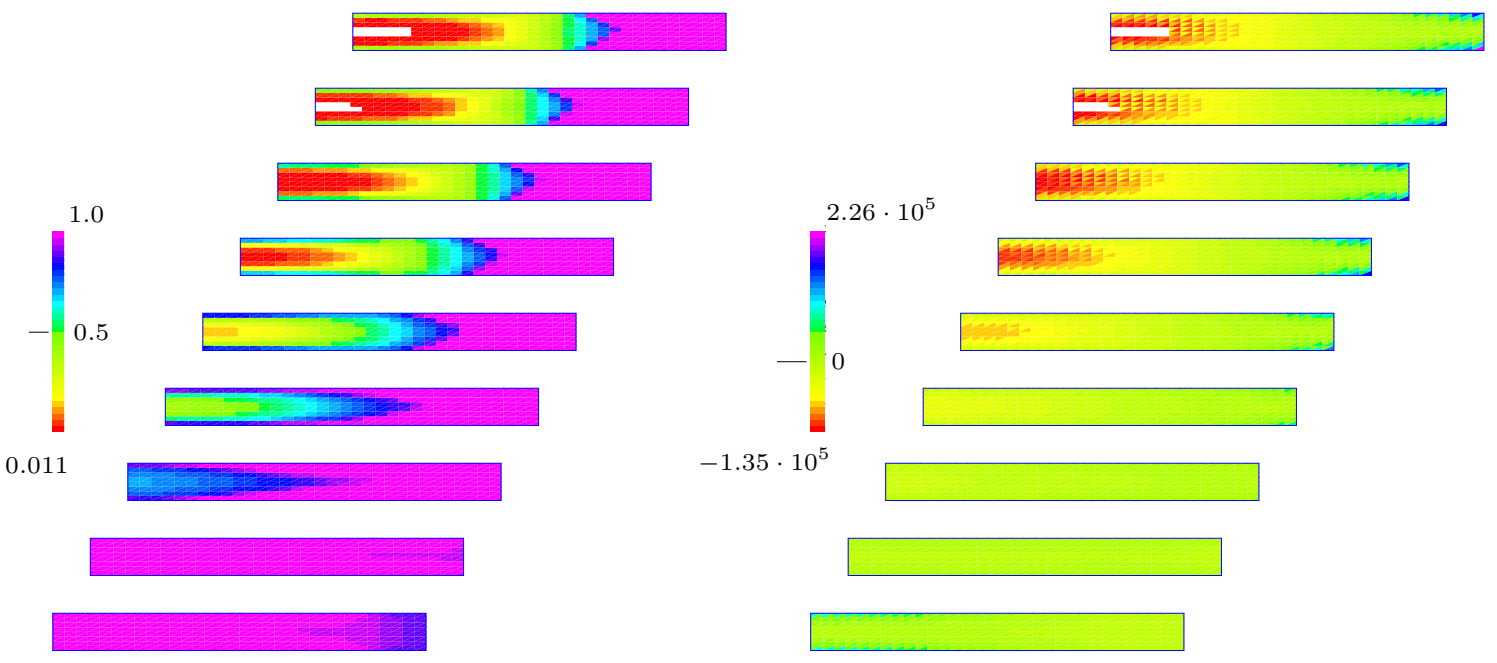

Figure 11: Clamped support, linear interpolation and $75 \%$ material. Left the optimized density distribution and right the corresponding distribution of $\mathrm{OC}$ values. Linear scales for density and $\mathrm{OC}$ values; white for $\rho_{e}<0.011$.

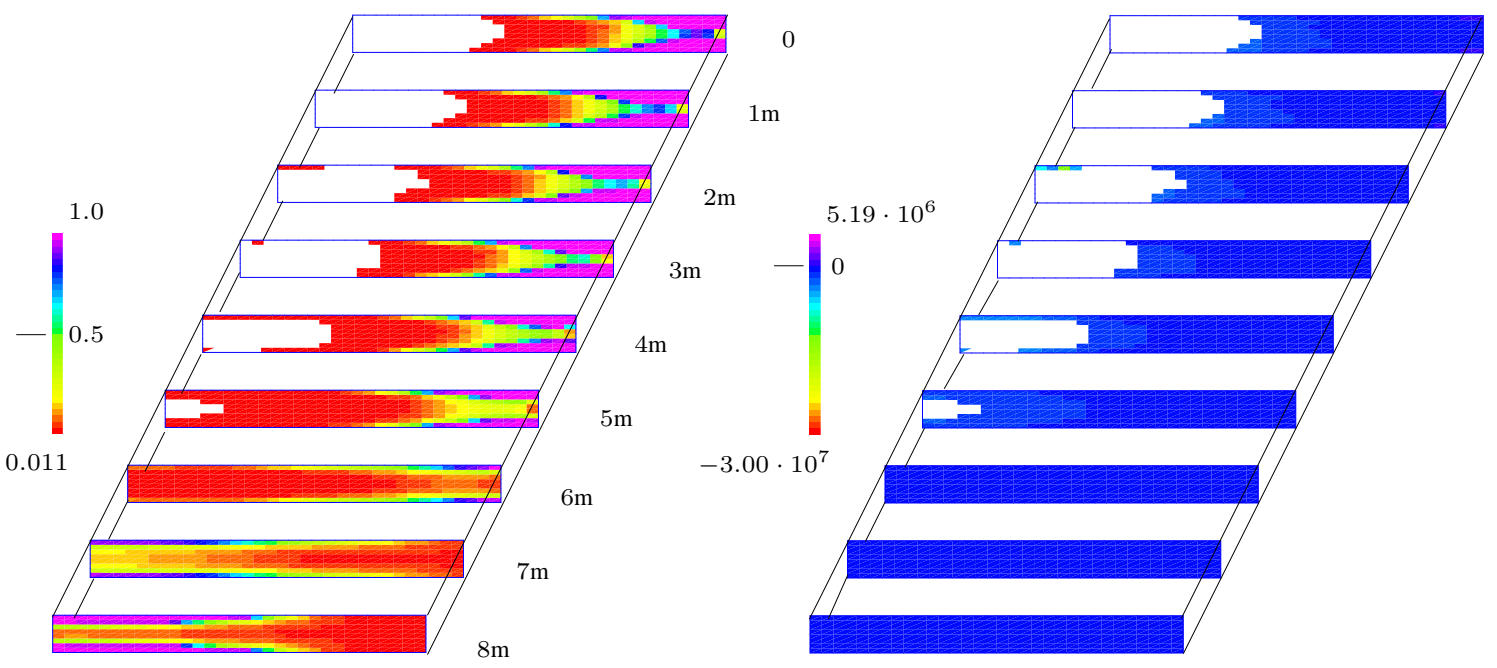

Figure 12: Clamped support, linear interpolation and 25\% material. Left the optimized density distribution and right the corresponding distribution of $\mathrm{OC}$ values. Linear scales for density and $\mathrm{OC}$ values; white for $\rho_{e}<0.011$. 


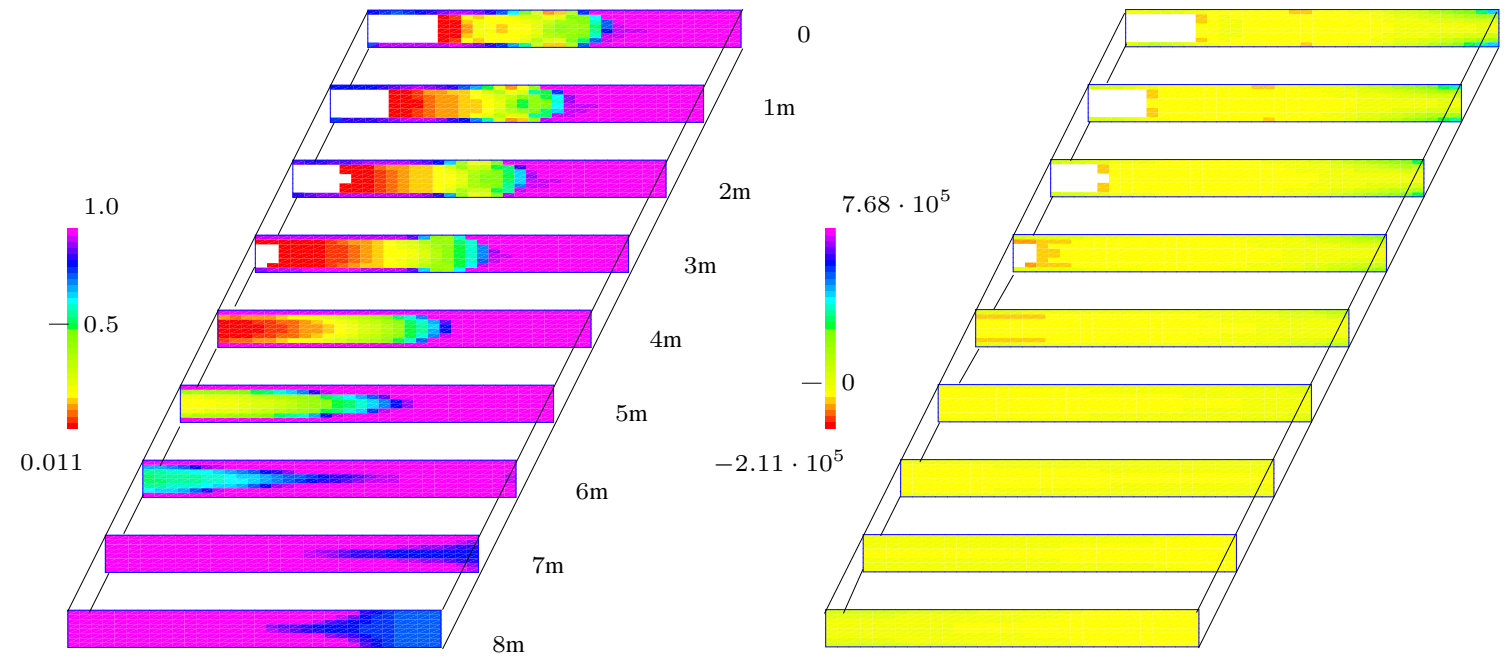

Figure 13: Clamped support, non-linear interpolation with $\kappa_{1}, \kappa_{0}=3,0.1$, and $75 \%$ material. Left the optimized density distribution and right the corresponding distribution of $\mathrm{OC}$ values. Linear scales for density and $\mathrm{OC}$ values; white for $\rho_{e}<0.011$.

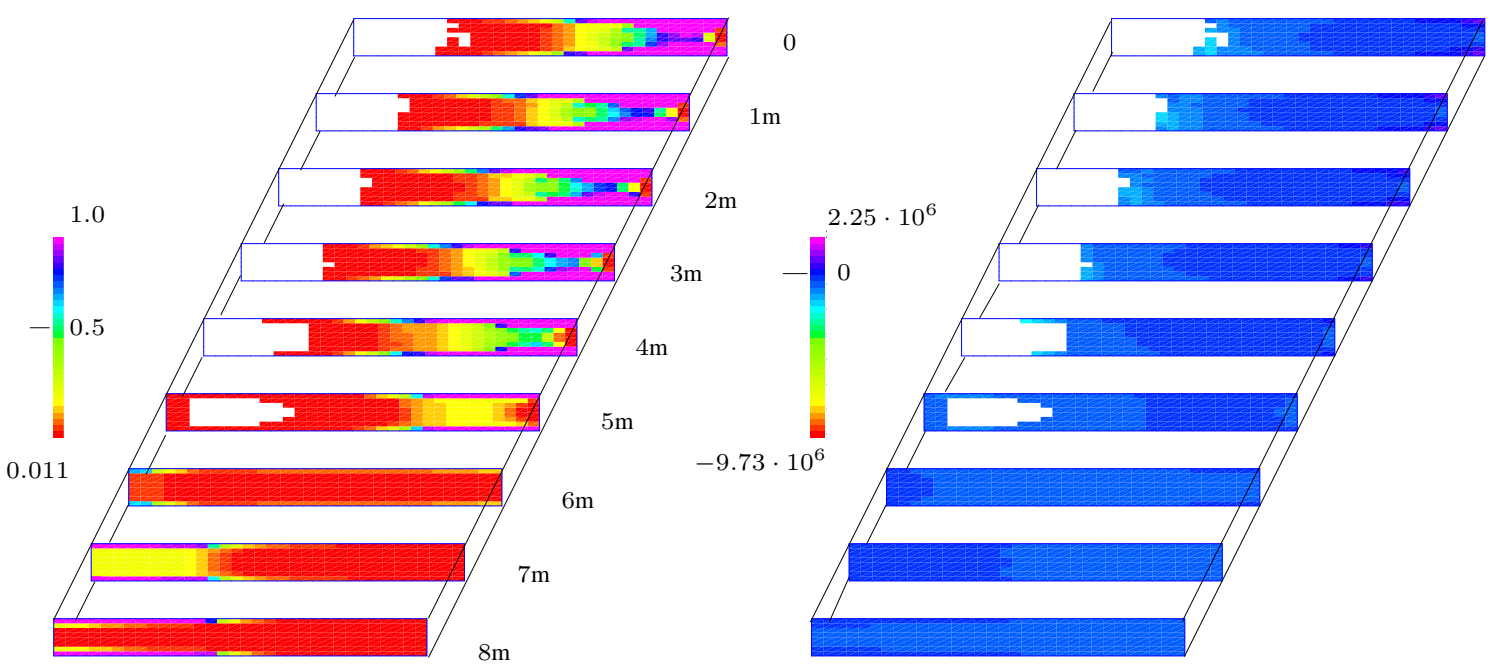

Figure 14: Clamped support, non-linear interpolation with $\kappa_{1}, \kappa_{0}=3,0.1$, and $25 \%$ material. Left the optimized density distribution and right the corresponding distribution of OC values. Linear scales for density and $\mathrm{OC}$ values; white for $\rho_{e}<0.011$. 
The comments to the results for clamped supports are as follows:

- In parallel to the presentations for simple support all Figures 11 - 14 show two distributions in the nine selected $x-y$, planes for $z=0,1,2,3,4,5,6,7,8 \mathrm{~m}$. The left part of each figure shows the optimized design after 15 redesigns, shown by its resulting $\rho_{e}$ distribution. The right part of each figure shows the OC values for the corresponding design.

- Figure 11 shows the final design after 6 redesigns with history in Figure 6a. A cavity is seen only for $z=0$ and 1 . For this design the distribution of values of OC is not completely uniform, but negative for all the active design parameters $\left(0.01<\rho_{e}<1\right)$, and therefore stopped after 6 iterations. All this indicate that optimization with $75 \%$ material is difficult.

- Figure 12 shows the final design with history in Figure 6b. Figure 12 is similar to Figure 11 above, except that the total amount of available material is now only $25 \%$. The extension of the cavity is larger and even a hole throughout the plate is seen. The resulting values of $\mathrm{OC}$ are rather uniformly distributed and close to zero.

- Figure 13 shows the final design with history in Figure 6c. Figure 13 is also similar to Figure 11, but now the stiffness interpolation is changed from linear to non-linear with the chosen parameters $\kappa_{1}, \kappa_{0}=3,0.1$. Cavities at the plate middle domain are seen. The resulting values of OC distribution are rather uniform and close to zero with positive values (green) at full material.

- Figure 14 shows the final design with history in Figure 6d. Figure 14 is similar to Figure 13 above, except that the total amount of available material is now only $25 \%$. With this less material available, the resulting design has an extended hole throughout the thickness in the middle of the plate. Rather uniform positive values of $\mathrm{OC}$ results. A seen in Figure $6 \mathrm{~d}$ the redesign is not converged, and more redesigns may give even more clear result. 


\section{References}

[1] R. Grandhi, Structural optimization with frequency constraints - a rewiev, AIAA Journal 31 (12) (1993) 2296-2303. 
[2] N. L. Pedersen, Maximization of eigenvalues using topology optimization, Struct. Multidisc. Optim. 20 (1) (2000) 2-11.

[3] J. Du, N. Olhoff, Topology optimization of freely vibrating continuum structures for maximum values of simple and multiple eigenfrequencies and frequency gaps, Struct. Multidisc. Optim. 34 (2007) 91-101.

[4] X. Huang, Z. H. Zuo, Y. M. Xie, Evolutionary topology optimization of vibrating continuum structures for natural frequencies, Computers and Structures 88 (2010) $357-364$.

[5] G. H. Yoon, Structural topology optimization for frequency response problem using model reduction schemes, Computer Methods in Applied Mechanics and Engineering 199 (2010) 1744-1763.

[6] L. Shu, M. Y. Wang, Z. Fang, Z. Ma, P. Wei, Level set based structural topology optimization for minimizing frequency response, J. of Sound and Vibration 330 (2011) 5820-5834.

[7] T. Liu, B. Li, S. Wang, L. Gao, Eigenvalue topology optimization of structures using a parameterized level set method, Struct. Multidisc. Optim. 50 (2014) 573591, note $=$.

[8] W. H. Wittrick, Rates of change of eigenvalues, with reference to buckling and vibration problems, J. Royal Aeronautical Soc. 66 (1962) 590-591.

[9] C. G. J. Jacobi, Uber ein leichtes verfahren die in der theorie der sacularstorungen vorkommenden gleichungen numerichen aufzulosen, Crelle's journal 30 (1846) 51-95.

[10] N. Olhoff, Optimal design of vibrating rectangular plates, Int. J. Solids Structures 10 (1974) 93-109.

[11] C. W. Bert, Optimal design of a composite-material plate to maximize its fundamental frequency, J. Sound and Vibration 50 (1977) 229-239. 
390 [12] K. T. Cheng, N. Olhoff, An investigation concerning optimal-design of solid elastic plates, Int. J. of Solids and Structures 17 (3) (1981) 305-323.

[13] P. Pedersen, N. L. Pedersen, A note on eigenfrequency sensitivities and structural eigenfrequency optimization based on local sub-domain frequencies, Struct. Multidisc. Optim. 49 (4) (2014) 559-568.

395 [14] P. Pedersen, Analytical stiffness matrices for tetrahedral elements, Computer Methods in Applied Mechanics and Engineering 196 (2006) 261-278. 